[Article]

\title{
基于纳米有机液体的高性能温差电池
}

\author{
孙浩宇鈇金欢唐桂华* \\ (西安交通大学能源与动力工程学院, 热流科学与工程教育部重点实验室, 西安 710049)
}

\begin{abstract}
摘要: 传统液体温差电池较低的热电转换性能一直无法得到有效改善, 亟需寻找新的热电转换机制来提升热 电转换效率。本文采用分子动力学(MD)方法, 数值模拟研究了不同温度下以不同配比的甘油-水为溶剂的氯 化钠溶液在碳纳米管(CNT)内离子、分子分布情况。结果表明: 离子、分子的分布受温度影响较大, 近壁面 净电荷、电势分布随温度升高出现明显的分层。根据模拟结果提出以 CNT 为电极材料, 甘油氯化钠溶液或 甘油水氯化钠溶液为电解质溶液组成温差电池。其热电转换性能远优于大多数温差电池, 同时温度适用范围 也显著增加。以多孔碳为电极材料, 甘油氯化钠溶液为电解质溶液组成的热电转换装置实验验证了可行性。
\end{abstract}

关键词: 分子动力学; 温差电池; 碳纳米管; 低温能源利用; 热电转换 中图分类号: 0646

\section{High-Performance Thermogalvanic Cell Based on Organic Nanofluids}

\author{
SUN Hao-Yu PU Jin-Huan TANG Gui-Hua*
}

(Key Laboratory of Thermal-fluid Science and Engineering of Ministry of Education, School of Energy and Power Engineering, Xi'an Jiaotong University, Xi'an 710049, P. R. China)

\begin{abstract}
The thermoelectric performance of traditional thermogalvanic cells is relatively low and a more efficient conversion mechanism is required. In this paper, the distribution of glycerol/glycerin in an aqueous sodium chloride solution in a carbon nanotube (CNT) is investigated by molecular dynamics (MD) simulation. The distributions of ions, molecule net charge, and electrical potential of the system are markedly affected by temperature. We propose a novel nanofluid thermoelectric conversion method based on the CNT and glycerol/ glycerin aqueous sodium chloride solution. The thermoelectric performance of the proposed system is much higher than that of most of current liquid thermogalvanic cells, and the application temperature range is also widened considerably. A preliminary thermal-to-electrical energy conversion experiment based on nanoporous carbon with mixtures of sodium chloride and glycerol is also conducted to qualitatively verify the numerical results.
\end{abstract}

Key Words: Molecular dynamics; Thermogalvanic cell; Carbon nanotube; Low-temperature energy utilization; Thermoelectric conversion

\section{1 引 言}

随着不可再生能源的日益枯竭以及能源需求 增大，低温能源(工业废水、汽车尾气、地热以及 太阳能等)的开发与利用受到越来越多的关注, 其 对改善能源环境问题及可持续发展具有深远的意 义。针对应用固体热电材料 ${ }^{1-5}$ 和斯特林发动机的
低温能源利用进行了大量的研究 ${ }^{6-9}$, 虽然取得了 较大的进步, 但是仍存在成本高、经济性低以及 可靠性差 ${ }^{10,11}$ 等问题。因此有学者将注意力转移到 液体温差电池上 ${ }^{12,13}$, 利用电解质溶液氧化还原对 以实现热电转换, 例如 $\mathrm{Cu}-\mathrm{CuSO}_{4}$ 和 $\mathrm{Zn}-\mathrm{ZnSO}_{4}$ 、 $\mathrm{FeCN}^{3-}-\mathrm{FeCN}^{4-}$ 以及 $\mathrm{Fe}^{3+}-\mathrm{Fe}^{2+}$ 等 ${ }^{14-18}$ 。然而依靠氧化

Received: May 13, 2016; Revised: June 27, 2016; Published online: June 28, 2016.

${ }^{*}$ Corresponding author. Email: ghtang@mail.xjtu.edu.cn; Tel: +86-29-82665319.

The project was supported by the National Natural Science Foundation of China (51576156).

国家自然科学基金(51576156)资助项目

(c) Editorial office of Acta Physico-Chimica Sinica 
还原对实现热电转换的经济性和热电转换性能仍 不能满足工业需求。为了突破液体温差电池的技 术瓶颈, 部分学者开始研究碳纳米管 $(\mathrm{CNT})$ 中电解 质溶液的性质, 并将其成功应用于低温热电转换 过程。 $\mathrm{Yu}$ 等 ${ }^{19}$ 以多孔碳为电极, 氯化钠水溶液为电 解质溶液组成的装置有效地将热能转换成电能。 $\mathrm{Hu}$ 等 ${ }^{20}$ 以 $\mathrm{CNT}$ 为电极, $\mathrm{FeCN}^{3-}-\mathrm{FeCN}^{4-}$ 水溶液为电 解质溶液制作成液体温差电池, 得到较高的电势 和热电转换效率。Chen 等 ${ }^{21-23}$ 研究发现当氯化钠水 溶液被限制在 CNT 内时, 溶液中的分子、离子分 布会发生各向异性变化, 导致 CNT 管壁产生电 势, 并且该电势大小受温度影响较大, 利用这个 机理能够实现热电转换且具有较高的转换效率; 同时 CNT 在热能、电能、机械能相互转换及海水 淡化 ${ }^{24,25}$ 等方面也具有优良的性能。但是水溶液存 在表面张力较大、不易浸入 CNT 以及稳定性差、 易受环境影响等缺点。

本文提出采用甘油氯化钠溶液和甘油水二元 混合氯化钠溶液作为电解质溶液, 可明显降低溶 液的表面张力, 使溶液更易于浸润 CNT, 稳定性 也相应地提高, 同时随着甘油在溶剂中的比例增 大, 相同温差下产生的电势也随之增大, 热电转 换性能显著增加。除此之外甘油与水相互混合增 加了热电转换的温度范围, 不再局限于 $0-100{ }^{\circ} \mathrm{C}$ 内, 显著增加了液体热电转换的适用范围。

\section{2 数值方法及物理模型}

采用分子动力学方法, 基于开源软件 $\mathrm{Lammps}^{26}$ 进行计算。物理模型如图 1 所示, 计算中 选用 CNT $(20,20)$ 扶手椅型碳纳米管并固定, 其半 径 $R=1.356 \mathrm{~nm}$, 计算长度 $l=9.838 \mathrm{~nm}$, 不同模拟 工况其电解质溶液组分见表 1 , 初始温度 $T=300$ $\mathrm{K}$, 压力为 $p=0.1 \mathrm{MPa}$ 。本文采用 Class 2 力场中 的 PCFF 力场(force field for polycarbonate) 以及宏观 正则系综(NVT系综, 其中 $N$ 代表体系中原子个 数, $V$ 代表体积, $T$ 代表温度), $\mathrm{CNT}$ 与溶液中分 子、离子之间的势能函数为 $E=\varepsilon_{i j}\left[2\left(\sigma_{i j} / r_{i j}\right)^{9}-3\left(\sigma_{i j} /\right.\right.$ $\left.r_{i j}\right)^{6}$, 交互作用参数采用 Lorentz-Berthelot 混合计 算法则, 截断半径设为 $1 \mathrm{~nm}$, 盒子轴向采用周期 性边界条件, 时间步长为 $1 \mathrm{fs}$ 。模拟 $1 \mathrm{~ns}$ 使溶液达 到平衡阶段, 平衡之后继续模拟 $1 \mathrm{~ns}$ 并进行数据输 出, 总模拟时间为 $2 \mathrm{~ns}$ 。

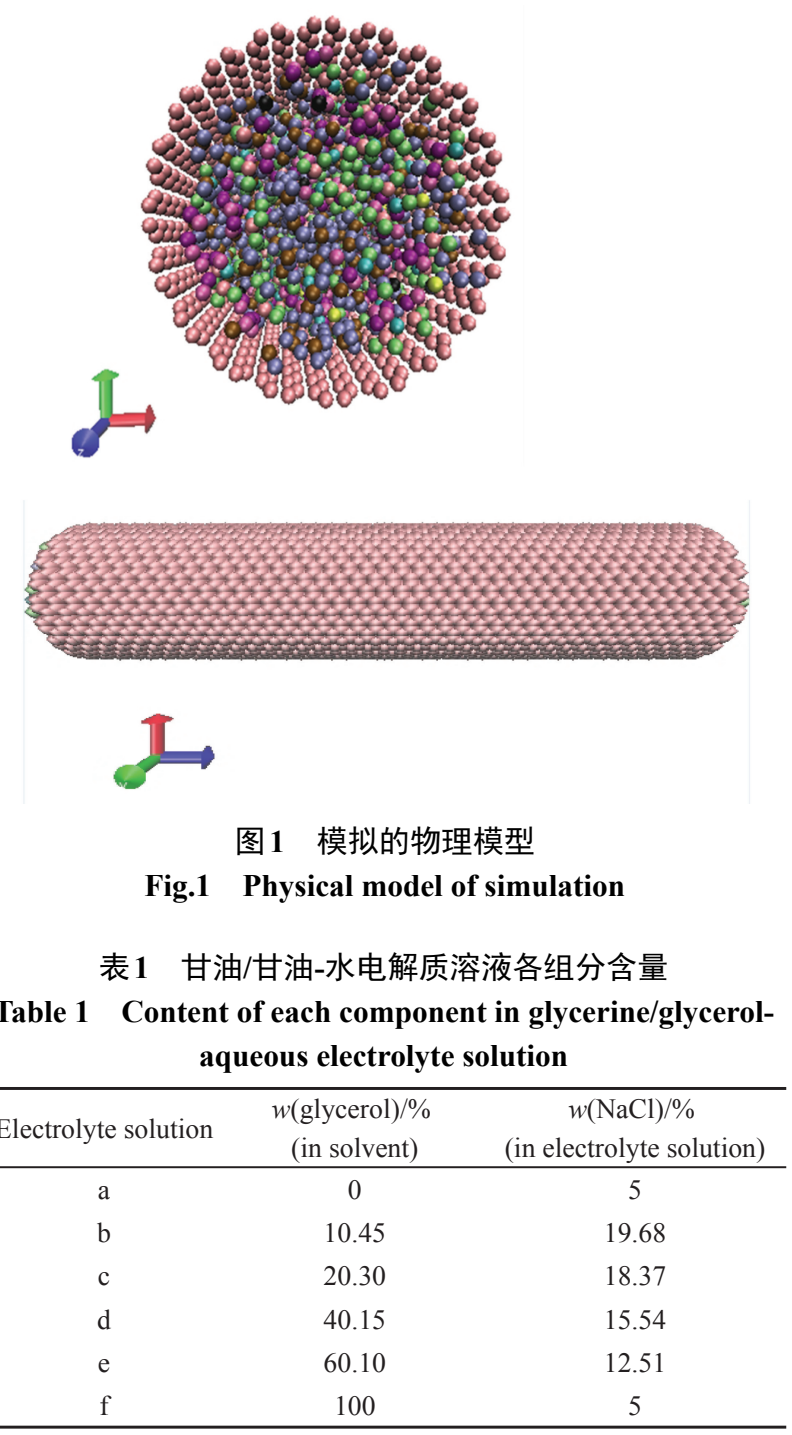

\section{3 结果与分析}

\subsection{CNT内分子和离子分布特性}

图 2 为温度 $300 \mathrm{~K}$ 时 $\mathrm{CNT}$ 内电解质溶液中离 子、分子的分布情况, 横坐标表示距离管径中心 的距离。图 2(A)为不同工况下 $\mathrm{Na}^{+}$分布情况, 图 2 (B) 为 $\mathrm{H}$ (甘油分子中与碳原子直接成健的氢原子) 分布情况。从图 2(A)中可知, 不同工况下近壁面 处 $\mathrm{Na}^{+}$具有相似分布特性, 从图 2(B) 可知, 不同工 况下近壁面处 $\mathrm{H}$ 的分布特性同样相似。事实上不 同工况下溶液中其他离子、分子均表现出相似的 分布特性, 本文不再赘述。由于不同工况下 CNT 内离子、分子分布特性相似, 下文选取溶剂中甘 油含量为 $60.10 \%$ 的氯化钠电解质溶液 (简称 $60.10 \%$ 甘油电解质溶液)进行分析。图 2(C) 为 $\mathrm{Na}^{+}$和 $\mathrm{Cl}^{-}$在 CNT 中径向分布情况; 图 2(D)为甘油分子和水分 子在 CNT 内分布情况, 左边纵坐标是甘油分子中 

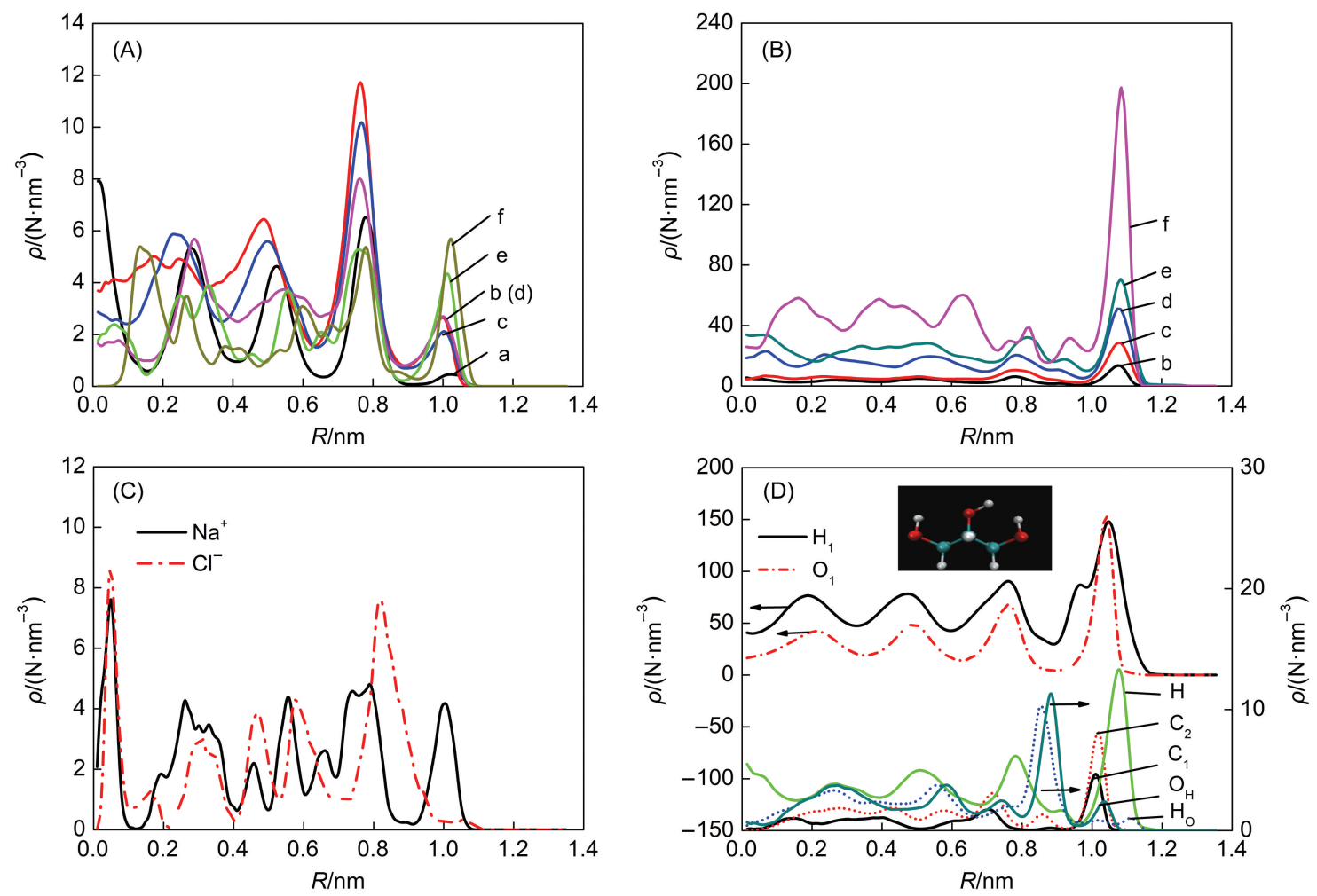

图 $2300 \mathrm{~K}$ 时 CNT 中电解质溶液离子、分子分布

Fig.2 Number density distribution of the ions and molecules in electrolyte solution of the CNT at $300 \mathrm{~K}$

(A) $\mathrm{Na}^{+}$, (B) $\mathrm{H}$ in glycerol, (C) and (D) are ions and solvent molecules for the electrolyte solution.

(a) no glycerol in solvent and $5 \% \mathrm{NaCl}$; (b) $10.45 \%$ glycerol in solvent and $19.68 \% \mathrm{NaCl}$; (c) $20.30 \%$ in solvent and $18.37 \% \mathrm{NaCl}$; (d) $40.15 \%$ glycerol in solvent and $15.54 \% \mathrm{NaCl}$; (e) $60.10 \%$ glycerol in solvent and $12.51 \% \mathrm{NaCl}$; (f) $100 \%$ glycerol and $5 \% \mathrm{NaCl} ; \mathrm{H}_{1}$ and $\mathrm{O}_{1}: \mathrm{H}_{\text {and }} \mathrm{O}$ in the water molecule; $\mathrm{C}_{1}, \mathrm{C}_{2}, \mathrm{H}, \mathrm{H}_{\mathrm{O}}, \mathrm{O}_{\mathrm{H}}: \mathrm{C}_{1}$ is the carbon atom at both end of the glycerol molecule, $\mathrm{C}_{2}$ is the carbon atom at the middle, $\mathrm{H}, \mathrm{H}_{\mathrm{O}}$ : are the hydrogen atoms directly bonded with the carbon and oxygen atoms, respectively, and $\mathrm{O}_{\mathrm{H}}$ is the oxygen in glycerol molecule.

各原子 $\left(\mathrm{C}_{1}\right.$ 表示分子链中间碳原子, $\mathrm{C}_{2}$ 表示碳链两 端的碳原子, $\mathrm{H}$ 表示直接与碳原子连接的氢原子, $\mathrm{H}_{\mathrm{O}}$ 表示与氧原子相连的氢原子， $\mathrm{O}_{\mathrm{H}}$ 表示与氢原子 相连的氧原子)密度在 CNT 中径向分布数值, 右边 纵坐标是水分子中各原子 $\left(\mathrm{H}_{1}\right.$ 表示水中的氢原子, $\mathrm{O}_{1}$ 表示水中的氧原子)密度在 CNT 中径向分布数 值。由图 3 可知, 阴阳离子以及分子密度在近壁面 处出现大的波动。溶液中的 $\mathrm{H}$ 原子距离壁面比 $\mathrm{O}$ 和 $\mathrm{C}$ 原子近, $\mathrm{Na}^{+}$距离壁面比 $\mathrm{Cl}^{-}$近。前者是因为 $\mathrm{H}$ 受 到 $\mathrm{CNT}$ 壁面的斥力比溶液中的 $\mathrm{O} 、 \mathrm{C}$ 弱; 后者主要 因为 $\mathrm{Na}^{+}$的半径比 $\mathrm{Cl}^{-}$的半径小, 所以 $\mathrm{Na}^{+}$受到来自 $\mathrm{H}$ 原子的引力较强, 受到 $\mathrm{O} 、 \mathrm{C}$ 原子的斥力较弱 ${ }^{22}$ 。

根据 Poisson 方程可以得到相应的电势 $\varphi$ 分 布, Poisson 方程如下

$$
\Delta^{2} \varphi(r)=-\rho_{\text {ne }}(r) /\left(\varepsilon_{0} \varepsilon\right)
$$

式中, $\varepsilon_{0}$ 表示真空介电常数; $\varepsilon$ 表示溶液的介电常 数; $\rho_{\text {ne }}(r)$ 表示净电荷密度, 可由下式获得:

$$
\rho_{\mathrm{ne}}(r)=\sum_{i=1}^{n} \rho_{i}(r) c_{i}
$$

式中, $n$ 表示溶液中粒子总数; $c_{i}$ 表示第 $i$ 个离子或 原子的电荷量。

根据二元无规混合物有效介电常数的计算公 式, 可得到甘油水氯化钠溶液的有效介电常数 $\varepsilon$, 其计算公式如下 ${ }^{27}$

$$
(1-\omega) \frac{\varepsilon-\varepsilon_{2}^{\mathrm{b}}}{2 \varepsilon_{2}^{\mathrm{b}}+\varepsilon}+\omega \frac{\varepsilon-\varepsilon_{1}^{\mathrm{b}}}{2 \varepsilon_{2}^{\mathrm{b}}+\varepsilon_{1}^{\mathrm{b}}}=0
$$

式中, $\omega$ 表示溶质质量分数; $\varepsilon_{1}^{b}=2 \omega /(3-\omega) \varepsilon_{1}$, $\varepsilon_{2}^{\mathrm{b}}=2(1-\omega) /(2+\omega) \varepsilon_{2}, \quad \varepsilon_{1}$ 表示氯化钠的介电常 数, $\varepsilon_{2}$ 表示甘油或甘油水混合溶液的介电常数, 如 表2 所示 ${ }^{28}$ 。

图 3(A)为 $T=300 \mathrm{~K}$ 时 $60.10 \%$ 甘油电解质溶液 在 CNT 内净电荷密度分布情况。可见, 在 CNT管 壁附近的净电荷密度波动较大, 这是由于 $\mathrm{CNT}$ 管 壁附近离子、原子密度分布不均匀, 并且相对集 中; 同时在 CNT 内近壁面净电荷密度分布随着温 度的升高而下降, 这是因为温度越高, 近壁面附 近的离子越易获得能量, 脱离近壁面的束缚, 导 致净电荷密度降低, 即温度越高, 近壁面内净电 

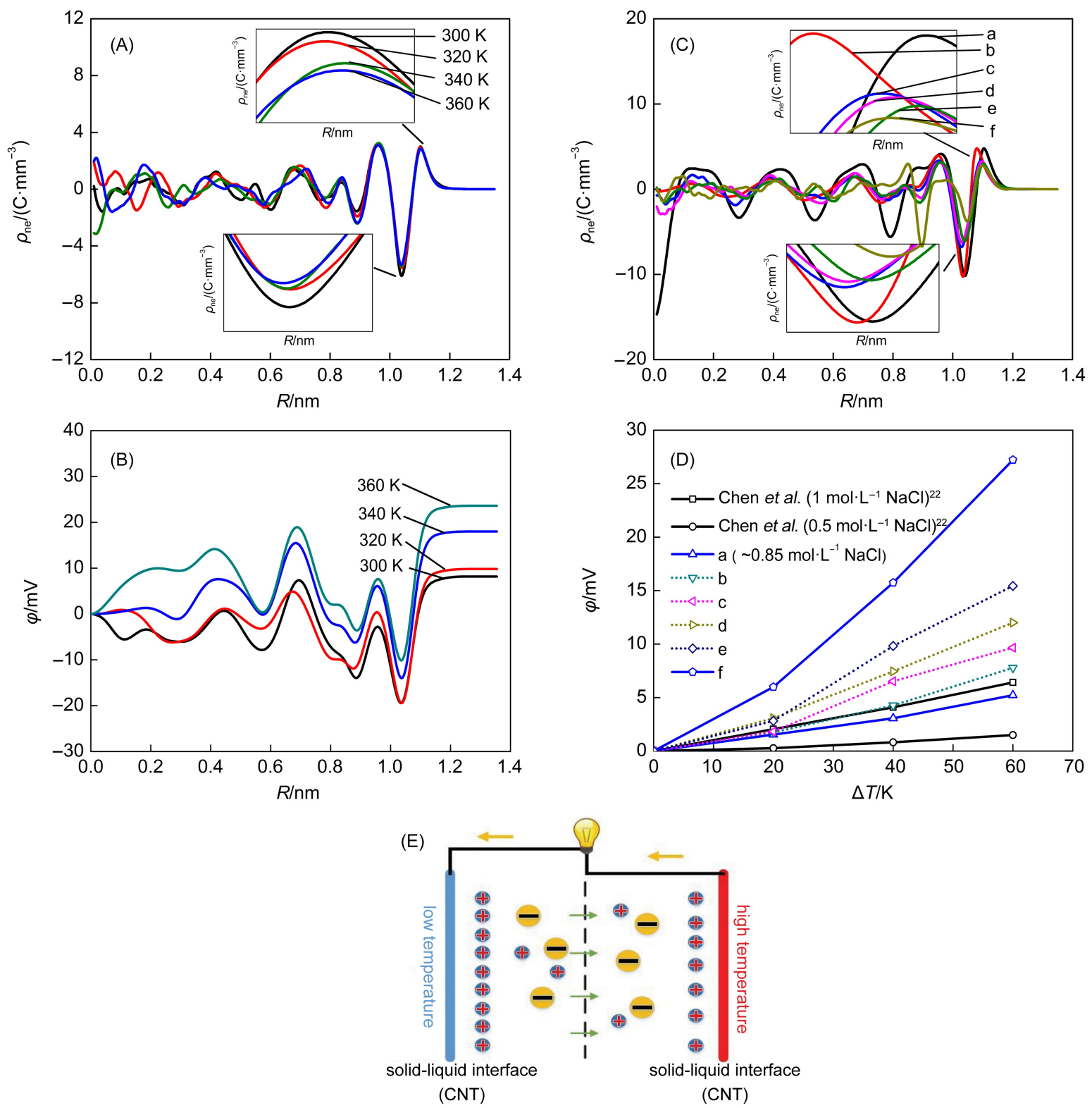

图 3 CNT 内电解质溶液净电荷密度及电势分布情况

Fig.3 Distribution of net charge and electric potential in the CNT

(A) net charge for electrolyte solution e, (B) electric potential for electrolyte solution e, (C) distribution of net charge at $T=300 \mathrm{~K}$,

(D) net output electric potential at different thermal differences $(\Delta T)$, (E) schematic of the potential difference generated between two liquid-CNT interfaces at a heat grade. The systems a-f are same as Fig.2. $\rho_{\text {ne: }}$ density of net charge, Chen: data from Chen's article ${ }^{21,22}$

荷密度越低。图3(B) 表示不同温度时 $60.10 \%$ 甘油 电解质溶液在 CNT 内电势分布。可见, 近壁面电 势随温度升高而增大, 这是由于 CNT 内近壁面净 电荷密度分布随温度升高而下降所导致。图 3(C) 为 $T=300 \mathrm{~K}$ 时不同工况 $\mathrm{CNT}$ 内净电荷密度分布。 观察到以纯水为溶剂的氯化钠溶液与以甘油为溶 剂的氯化钠溶液相比波动的峰值比较平均, 纯甘 油氯化钠溶液的净电荷主要集中在近壁面附近; 不同比例的甘油水混合饱和氯化钠溶液净电荷密
度分布具有相同的波动规律, 同时随甘油含量的 增加和氯化钠含量的减少而降低。图 3(D) 给出了 各工况处于不同温度 CNT 相互连接(如图 3(E))输出 电势 $\varphi$ 随温差 $\Delta T$ 变化, 同时与 $\mathrm{Chen}$ 等 ${ }^{22}$ 模拟结论 进行对比。可知, 输出电势 $\varphi$ 随温差 $\Delta T$ 增大而增 加; 纯甘油氯化钠溶液的输出电势 $\varphi$ 明显大于纯水 氯化钠溶液的输出电势; 甘油水二元混合饱和氯 化钠溶液的输出电势 $\varphi$ 随甘油含量的增加及氯化钠 含量的减少而升高, 而根据 Chen 等 ${ }^{22}$ 研究, 纯水 
表 2 不同比例的甘油和水混合物在不同温度下 介电常数 $\left(\varepsilon_{2}\right)$

Table 2 Dielectric constants $\left(\varepsilon_{2}\right)$ of glycerol-water solutions under different temperatures

\begin{tabular}{cccccc}
\hline \multirow{2}{*}{$w($ glycerol $) / \%$} & \multicolumn{5}{c}{$\varepsilon_{2}$} \\
\cline { 2 - 6 } & $25^{\circ} \mathrm{C}$ & $40{ }^{\circ} \mathrm{C}$ & $60{ }^{\circ} \mathrm{C}$ & $80{ }^{\circ} \mathrm{C}$ & $100{ }^{\circ} \mathrm{C}$ \\
\hline 10 & 75.7 & 70.41 & 63.98 & 58.31 & - \\
20 & 72.9 & 67.70 & 61.56 & 56.01 & - \\
30 & 70.0 & 64.87 & 58.97 & 53.65 & - \\
40 & 67.1 & 62.03 & 56.24 & 51.17 & - \\
50 & 64.0 & 59.55 & 53.36 & 48.52 & - \\
60 & 60.0 & 55.48 & 50.17 & 49.39 & 41.08 \\
70 & 55.6 & 51.41 & 46.33 & 41.90 & 38.07 \\
100 & 40.1 & 37.30 & 33.82 & 30.63 & 27.88 \\
\hline
\end{tabular}

氯化钠溶液的输出电势 $\varphi$ 应该随纯水氯化钠浓度减 少而降低, 所以输出电势 $\varphi$ 逐渐增大主要是由甘油 含量增加所导致。由图 3(D)可知, 本文模拟的氯 化钠水溶液的结果与 Chen 等模拟结论相符合。

\section{2 热电转换效率与 Seebeck 系数}

热电转换效率公式为 $\eta=W_{\text {input }} / W_{\text {output }}$, 其中 $W_{\text {input }}$ 表示输入功, $W_{\text {ouput }}$ 表示输出功。液体温差电池热 电转换过程类似于电容放电, 输出功的过程也相 当于电极放电做功, 所以输出功 $W_{\text {output }}$ 可由下式给 出 ${ }^{22}$

$$
W_{\text {output }}=1 / 2 \Delta Q U
$$

其中 $U=Q / C_{\mathrm{T}}$, 则 :

$$
W_{\text {output }}=\Delta Q \times Q /\left(2 C_{\mathrm{T}}\right)
$$

式中 $C_{\mathrm{T}}$ 表示电容, $\Delta Q$ 表示最大电荷变量, $Q$ 为近 壁面总的初始电荷量。由于电容是由阴、阳极电 容组成, 故:

$$
1 / C_{\mathrm{T}}=1 / C_{\mathrm{a}}+1 / C_{\mathrm{c}}
$$

式中, $C_{\mathrm{a}}$ 表示阳极电容; $C_{\mathrm{c}}$ 表示阴极电容 ${ }^{29}$ 。本文 所选取的 CNT 属于介孔 $(2 \mathrm{~nm}<d<50 \mathrm{~nm})$, 所以 电容 $C=2 \pi \varepsilon \varepsilon_{0} l / \ln (b /(b-d)), \quad \varepsilon_{0}=8.854 \times 10^{-12} \mathrm{~F}$. $\mathrm{m}^{-1} ; l$ 是纳米管长度; $b$ 是碳管半径; $d$ 是壁面与 近壁面离子的距离, 如图4所示。

$$
W_{\text {input }}=\sum_{1}^{n} C_{\mathrm{i}} \Delta T
$$$$
\text { 系统的输入功 } W_{\text {input }} \text { 为: }
$$

式中, $C_{\mathrm{i}}$ 表示各原子/离子的比热容。对于甘油分 子、水分子、 $\mathrm{C} 、 \mathrm{Na}^{+}$和 $\mathrm{Cl}^{-}$的比热分别为 202.58 、 $74.54 、 8.52 、 27.60$ 和 $33.95 \mathrm{~J} \cdot \mathrm{mol}^{-1}$ 。为了更好地 描述系统的性能, 通常采用其相对于卡诺循环的 效率 $\eta_{\mathrm{r}}$ :

$$
\eta_{\mathrm{r}}=\eta / \eta_{\mathrm{c}}=\left(W_{\text {input }} / W_{\text {output }}\right) /\left(\Delta T / T_{\text {hot }}\right)
$$

Seebeck 系数计算公式为 $\alpha=\partial V / \partial T, V$ 表示电势, $T$ 表示温度; $\alpha$ 表示电势随温度变化快慢。

图 5(A) 为不同工况下热电转换 Seebeck 系数 $\alpha$ 与温差 $\Delta T$ 的关系, 发现纯甘油氯化钠溶液和甘油 水氯化钠溶液热电转换的 Seebeck 系数随温差 $\Delta T$ 增 加而增加, 而纯水氯化钠溶液的 Seebeck 系数 $\alpha$ 随 温差 $\Delta T$ 升高变化不明显；当温差 $\Delta T=40,60 \mathrm{~K}$ 时, 甘油水氯化钠溶液的 $\alpha$ 随甘油含量的增加而增大。 这说明在较大温差时, 随着甘油含量的增加 CNT 内电解质溶液对温度的敏感性越高。图 5(B) 表示 不同工况下热电转换效率 $\eta$ 随温差变化, 发现相同 氯化钠浓度时, 纯甘油氯化钠电解质溶液的效率 $\eta$ 要远高于纯水氯化钠溶液; 甘油水二元混合饱和 氯化钠溶液时, 热电转换效率随甘油含量的增加 (氯化钠浓度减小)而增加, 根据 Chen 等 ${ }^{22}$ 研究可 知, 氯化钠浓度减小, 热电转换效率减少, 所以 甘油含量的增加是引起热电转换效率升高的主要 原因。图 5(C) 为不同工况下该机制相对于卡诺循 环的热电转换效率 $\eta_{\mathrm{r}}$ 随温差的变化。纯甘油氯化钠 溶液和甘油水氯化钠溶液热电转换 $\eta_{\mathrm{r}}$ 均随温差增加 而减小, 说明该机制的热电转换效率温差越小越 接近卡诺循环效率，温差越大越偏离卡诺循环。

综上所述, 在 CNT 内氯化钠浓度相同时氯化 钠甘油溶液比氯化钠水溶液性能更佳; 甘油水二 元混合饱和氯化钠电解质溶液的热电转换性能在 温差较小、甘油含量较多情况下更加优异。本文 热电转换机制在 $\Delta T=60 \mathrm{~K}$ 时, 相对于卡诺循环 的热电转换效率 $\eta_{\mathrm{r}}$ 高达 $11.30 \%$ ，而大多数液体 温差电池相对于卡诺循环的热电转换效率 $\eta_{\mathrm{r}}$ 仅为 $\sim 1.40 \%{ }^{13,16,20,30}$ 。

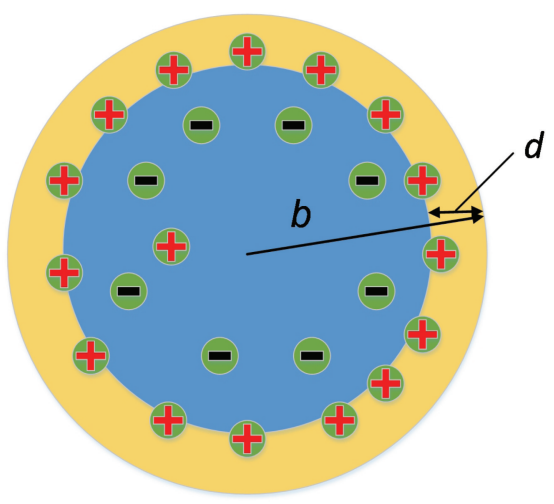

图 4 电解质溶液中离子在介孔内分布示意图

Fig.4 Schematic of distribution of ions in the mesopore in electrolyte solution 

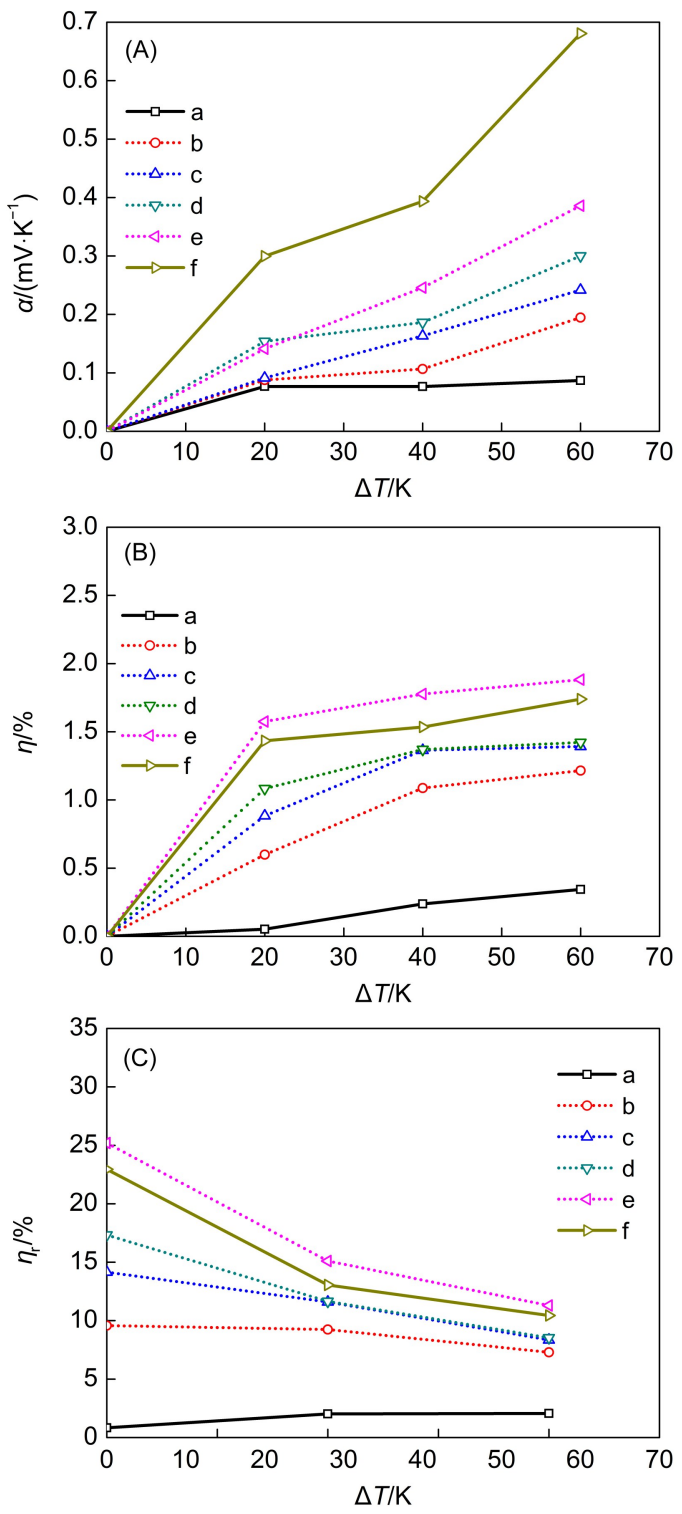

图 5 不同浓度甘油溶剂电解质溶液的 $\alpha 、 \eta 、 \eta_{\mathrm{r}}$ 随温差 $\Delta \boldsymbol{T}$ 的变化情况

Fig.5 Changes of $\alpha, \eta$, and $\eta_{\mathrm{r}}$ with $\Delta T$ in different electrolyte solutions

(A) Seebeck coefficient, (B) thermoelectric conversion efficiency,

(C) thermoelectric conversion efficiency of Carnot efficiency. The systems a- $\mathrm{f}$ are same as Fig.2.

\section{3 实验验证}

当电解质溶液限制在 CNT 中, 由于阴阳离子 径向分布的差异, 以及溶剂分子中各原子分布不 同而产生偶极子的有序取向 ${ }^{31,32}$, 会导致其内部电 荷分布不均匀, 产生净电荷及电势。通过模拟分 析可知, CNT 内净电荷及电势分布受温度影响较 大, 由此将处于不同温度下的 CNT 连接形成回 路, 能够输出电势 $\varphi$, 实现热能向电能的转换。本 实验采用碳纳米多孔材料炭黑代替 CNT 验证该机
制可行性。

实验以工业炭黑(纯度 $>99.5 \%$, 天津金秋实 化工有限公司)为电极材料, 甘油水-氯化钠溶液 (甘油纯度 $>99 \%$, 氯化钠纯度 $>99.5 \%$ ) 为电解质 溶液组成热电转换装置。首先, 将炭黑用丙酮(纯 度 $>98.5 \%$ ) 清洗 $4 \mathrm{~h}$ 晾干; 然后将其压制成直径为 $10 \mathrm{~mm}$ 的圆盘电极, 每个圆盘电极的质量电极大约 为 $90 \mathrm{mg}$; 最后用导电银胶将电极与边长为 $5 \mathrm{~mm}$ 的铜䈃(作为集流器)连接并放入 DZ-3AII 真空干燥 箱(奥环电子产品有限公司)内, $120^{\circ} \mathrm{C}$ 烘干 $4 \mathrm{~h}$, 并将连接集流器侧的电极表面进行防水处理。实 验系统示意图如图 6所示。实验采用 F26-ME 恒温 水浴系统(德国 Julabo 公司) 保证两端温度恒定, 冷、热两端采用直径为 $5 \mathrm{~mm}$ 的盐桥连接。冷端温 度保持 $25^{\circ} \mathrm{C}$ 不变, 通过调节高温端恒温水浴的温 度来改变温差 $\Delta T$ 。用 Keithley 2700 数据采集器(美 国 Keithley 公司)采集温度 $T$ 以及不同温差 $\Delta T$ 下输 出的电压 $\varphi$ 。

通过实验得到甘油氯化钠电解质溶液温差电 池电势随温差变化情况, 如图 7 所示。可知, 电势 随着温差 $\Delta T$ 的增大而增大, 相同氯化钠浓度下氯

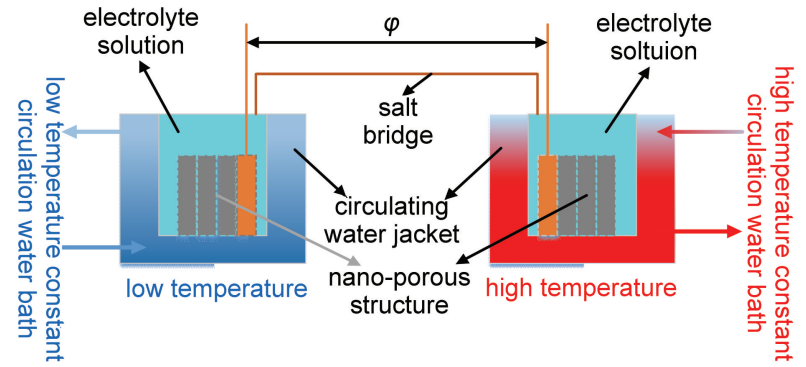

图 6 纳米有机液体温差电池实验示意图

Fig.6 Schematic of the thermogalvanic cell based on organic nanofluids

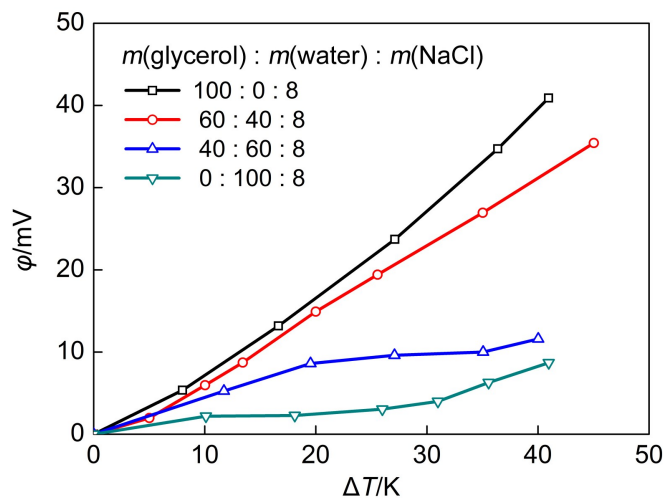

图 7 电极两端电势随温差 $\Delta \boldsymbol{T}$ 变化情况

Fig.7 Output electric potential between two electrodes 
化钠甘油电解质溶液产生的电势高于氯化钠水电 解质溶液, 甘油/水混合氯化钠电解质溶液电势随 甘油含量的增加而增大, 与数值模拟结果一致。 同时发现, 当氯化钠浓度相同时, 溶剂中甘油含 量下降到 $40 \%$, 电势随温差 $\Delta T$ 升高而增加的量明 显下降, 与纯水氯化钠电解质溶液接近, 说明此 时电解质溶液中甘油对离子分布的影响下降, 本 文认为是因为氯化钠在水中的溶解度大于在甘油 中的溶解度导致。实验结果与数值模拟结果定量 上的差异是由于实验所用的多孔电极的孔径及导 电、导热性能和表面粗楉度均与碳纳米管存在差 异。

\section{4 结 论}

通过 MD 方法模拟 CNT- 甘油氯化钠溶液及 CNT-甘油水氯化钠溶液的纳米有机液体温差电池 的热电转换过程以及实验验证研究, 获得以下主 要结论:

(1) CNT 内溶液中的离子、分子分布不均, 近 壁面净电荷密度 $\rho_{\text {ne }}$ 随温度升高而下降, 近壁面电 势 $\varphi$ 随温差增加而增加。

(2) CNT 内近壁面净电荷密度 $\rho_{\mathrm{ne}}$ 随甘油含量增 加以及氯化钠含量的降低而降低。

(3) 将处于不同温度下的 CNT 相互连接形成回 路能够实现热能向电能的转换, 热电转换 Seebeck 系数及热电转换效率随温差的增加而增大, 甘油/ 水二元混合饱和氯化钠溶液时, 热电转换效率随 甘油含量增加而增加; 低温差时具有较高的 $\eta_{\mathrm{r}}$, 其 性能优于传统温差电池。

(4) 实验验证发现, 氯化钠浓度相同时, 氯化 钠甘油溶液热电转换性能要优于氯化钠水溶液, 甘油水氯化钠电解质溶液电势随甘油含量增加而 增加。

另外, CNT 孔径、氯化钠的浓度、初始温度 等均会影响热电转换性能。该热电转换机制具有 以下优点：电极与溶液不存在化学反应, 所以对 电极材料的损耗极低, 碳的排放量为零; 电极材 料可由工业炭黑代替，成本低；不同比例甘油水 混合溶剂的凝固点和沸点不再局限于 0 和 $100^{\circ} \mathrm{C}$, 热电转换温度范围显著增加。该热电转换机制具 有较高的转换效率，对将来的低温热电转换研究 具有指导意义。

\section{References}

(1) Heremans, J. P.; Dresselhaus, M. S.; Bell, L. E.; Morelli, D. T. Nat. Nanotech. 2013, 8, 471. doi: 10.1038/nnano.2013.129

(2) Tritt, T. M. Annu. Rev. Mater. Res. 2011, 41, 433. doi: 10.1146/ annurev-matsci-062910-100453

(3) Snyder, J. G.; Toberer, E. S. Nat. Mater. 2008, 7, 105. doi: $10.1038 /$ nmat2090

(4) Bell, L. E. Science 2008, 321, 1457. doi: 10.1126/ science. 1158899

(5) Poudel, B.; Hao, Q.; Ma, Y.; Lan, Y. C.; Minnich, A.; Yu, B.; Yan, X.; Wang, D. Z.; Muto, A.; Vashaee, D.; Chen, X. Y.; Liu, J. M.; Dresselhaus, M. S.; Chen, G.; Ren, Z. F. Science 2008, 320, 634. doi: 10.1126/science. 1156446

(6) Kongtragool, B.; Wongwises, S. Renew. Sust. Energy Rev. 2003, 7, 131. doi: 10.1016/S1364-0321(02)00053-9

(7) Kong, X. O.; Wang, R. Z.; Huang, X. H. Energ. Convers. Manage. 2004, 45, 433. doi:10.1016/j.enconman.2003.09.009

(8) Kongtragool, B.; Wongwises, S. Renew. Energy 2007, 32, 547. doi: 10.1016/j.renene.2006.03.003

(9) Thombare, D. G.; Verma, S. K. Renew. Sust. Energy Rev. 2008 , 12, 1. doi: 10.1016/j.rser.2006.07.001

(10) Vining, C. B. Nat. Mater. 2009, 8, 83. doi: 10.1038/nmat2361

(11) Mancini, T.; Heller, P.; Butler, B.; Osborn, B.; Schiel, W.; Goldberg, V.; Buck, R.; Diver, R.; Andraka, C.; Moreno, J. ASME J. Sol. Energy 2003, 125, 135. doi: 10.1115/1.1562634

(12) Kuzminskii, Y. V.; Zasukha, V. A.; Kuzminskaya, G. Y. J. Power Sources 1994, 52, 231. doi: 10.1016/0378-7753(94) 02015-9

(13) Quickenden, T. I.; Mua, Y. J. Electrochem. Soc. 1995, 142 , 3985. doi: 10.1149/1.2048446

(14) Bouty, E. J. Phys. 1880, 9, 229. doi: 10.1051/jphystap: 018800090022901

(15) Debethune, A, J.; Licht. T. S.; Swendeman, N. J. Electrochem. Soc. 1959, 106, 616. doi:10.1149/1.2427448

(16) Gunawa, A.; Lin, C. H.; Buttry, D. A.; Mujica, V.; Taylor, R. A.; Prasher, R. S.; Phelan, P. E. Nano. Micro. Therm. 2013, 17 (4), 304. doi: 10.1080/15567265.2013.776149

(17) Quickenden, T. I.; Vernon, C. F. Sol. Energy 1986, 36, 63. doi: 10.1016/0038-092X(86)90061-7

(18) Kang, T. J.; Fang, S.; Kozlov, M. E.; Haines, C. S.; Li, N.; Kim, Y. H.; Chen, Y. S.; Baughman, R. H. Adv. Funct. Mater. 2012, 22, 477. doi: 10.1002/adfm.201101639

(19) Yu, Q.; Punyamurtual, V. K.; Han, A.; Lim, H. J. Power Sources 2008, 183, 403. doi:10.1016/j.jpowsour.2008.05.008

(20) Hu, R. C.; Cola, B. A.; Haram, N.; Barisci, J. N.; Lee, S.; Stoughton, S.; Wallace, G.; Too,C.; Thomas, M.; Gestos, A.; Cruz, M. E. D.; Ferraris, J. P.; Zakhidow, A. A.; Baughman, R. H. Nano Lett. 2010, 10, 838. doi: 10.1021/n1903267n

(21) Chen, X.; Xu, B. X.; Liu, L. Appl. Mech. Rev. 2014, 66, 050803. doi: $10.1115 / 1.4026913$

(22) Xu, B. X.; Liu, L.; Lim, H.; Qiao, Y.; Chen, X. Nano Energy 
2012, 1, 805. doi: 10.1016/j.nanoen.2012.07.013

(23) Xu, B. X.; Yu, Q.; Park, T.; Tak, M.; Zhou, Q. L.; Chen, X. Energy Environ. Sci. 2011, 4, 3632. doi: 10.1039/c1ee01405f

(24) Li, Q.; Yang, D. F.; Wang, J. H.; Wu, Q.; Liu, Q. Z. Acta Phys. -Chim. Sin. 2016, 32 (3), 691. [李 清, 杨登峰, 王建花, 武 琪, 刘清芝. 物理化学学报, 2016, 32 (3), 691.] doi: 10.3866/PKU.W HXB201512182

(25) Shen, Z. L.; He, G. H.; Zhang, N.; Hao, C. Acta Phys. -Chim. Sin. 2015, 31 (6), 1025. [谌庄琳, 贺高红, 张 宁, 郝 策. 物理 化学学报, 2015, 31 (6), 1025.] doi: 10.3866/PKU. WHXB201504141

(26) Plimpton, S. J. Comput. Phys. 1995, 117, 1. doi: 10.1006/ jcph.1995.1039

(27) Xue, Q. Z.; Li, W. Y. J. University Petroleum 1999, 23 (4), 102.
[薛庆忠, 李文瀛. 石油大学学报, 1999, 23 (4), 102.] doi: 10005870(1999)04-0102-03

(28) Association, G. P. Physical Properties of Glycerine and Its Solutions; Glycerine Producers' Association: New York 1963; p 17.

(29) Zhang, L. L.; Zhao, X. S. Chem. Soc. Rev. 2009, 38, 2520. doi: $10.1039 / \mathrm{b} 813846 \mathrm{j}$

(30) Mua, Y.; Quickenden, T. I. J. Electrochem. Soc. 1996, 143 (8), 2558. doi: 10.1149/1. 1837047

(31) Mann, D. J.; Halls, M. D. Phys. Rev. Lett. 2003, 90, 195503. doi: 10.1103/PhysRevLett.90.195503

(32) Zhu, F.; Schulten, K. Biophys. J. 2003, 85, 236. doi: 10.1016/ S0006-3495(03)74469-5 